\title{
Theory Vision
}

\author{
Gavin P. Salam* \\ Rudolf Peierls Centre for Theoretical Physics, Clarendon Laboratory, Oxford OX1 3PU, UK, \\ All Souls College, Oxford OX1 4AL, UK, \\ CERN, Theoretical Physics Department, CH-1211 Geneva 23, Switzerland. \\ E-mail: gavin.salam@physics.ox.ac.uk
}

Particle physics is sometimes described as going through a crisis, notably because of the continued lack of discovery of physics beyond the Standard Model, despite the LHC having operated at close to maximal energy for some years now. Here, I argue that we should not underestimate the significance of recent progress and future prospects in the Higgs sector of the Standard Model. This is especially the case for the Yukawa interactions and the structure of the Higgs potential, both of which are unlike any sector that has been established and stress-tested before in particle physics. Other topics that I touch on include the still substantial scope for increasing the reach of searches at LHC, the increasing role of precision in hadron-collider physics and the rich interplay that is developing between heavy-ion and proton-proton physics.

Sixth Annual Conference on Large Hadron Collider Physics (LHCP2018)

4-9 June 2018

Bologna, Italy

* On leave from CNRS, UMR 7589, LPTHE, F-75005, Paris, France. 


\section{Introduction}

A typical "Vision" talk often addresses the big, unanswered questions, such as the nature of dark matter and dark energy, questions about fine tuning and the origin of the matter-antimatter asymmetry of the universe. It is much less likely to discuss the Standard Model other than, sometimes, to briefly state that the Standard Model is now complete.

Searching for answers to big unanswered questions is vitally important. In doing so, however, we should not forget the importance of big answerable questions and the issue of how we go about answering them. That, for the most part, is what this talk is about.

The starting point is to examine the Standard Model and ask what we genuinely know about it, i.e. to what extent it is as complete as is often stated. The Standard Model, as a theoretical construction, is not just about its particle content but, just as importantly, about a set of interactions. Those interactions are summarised on a famous CERN T-shirt in the following compact form:

$$
\mathcal{L}=-\frac{1}{4} F_{\mu \nu} F^{\mu v}+i \bar{\psi} \not D \psi+\left|D_{\mu} \phi\right|^{2}+\psi_{i} y_{i j} \psi_{j} \phi+\text { h.c. }-V(\phi) .
$$

That T-shirt comes with an accompanying card that says "this equation neatly sums up our current understanding of fundamental particles and forces". What is the meaning of "understanding" in this context? Is it knowledge? Is it an assumption? Depending on the part of the Lagrangian that you examine, the answer differs.

The pure gauge $F_{\mu \nu} F^{\mu \nu}$ and matter-gauge $i \bar{\psi} D D \psi$ terms are arguably knowledge. The $i \bar{\psi} D \bar{\psi}$ term encodes interactions such as $e e \gamma, e v W, q q g, q q Z$ that have been established to high accuracy in $e^{+} e^{-}$, DIS and hadron-collider experiments. In terms of interactions, $F_{\mu \nu} F^{\mu v}$ term encodes in particular triple-gauge interactions such as $g g g$ and $Z W W$ that have similarly been probed experimentally at a range of past and present colliders. When one thinks of the immense success of the Standard Model over the past 40 years, it is in most cases a success involving the gauge sector, a sector whose history dates back, in some sense, to Maxwell's equations. If we measure yet another of the myriad individual contributions to these structures, considering all possible flavour and gauge-boson combinations, it is an important contribution to validating the overall framework of the Standard Model, but it is perhaps not surprising if we are underwhelmed by the success, yet again, of a test of the Standard Model.

The rest of Eq. (1) deals with the Higgs sector of the Standard Model, which was discussed extensively during LHCP (see for the example the talks by Grefe, Ortona, Sanz and Vryonidou [1, $2,3,4]$ ). I will spend quite some time discussing that in the next section. That will be followed by a briefer discussion of searches and anomalies, precision Standard Model theory and heavy-ion collisions.

\section{The Higgs sector of the Standard Model}

Of the remaining terms in Eq. (1), one of them, $\left|D_{\mu} \phi\right|^{2}$, which again involves a gauge interaction, but now with the Higgs field, can also be argued to be knowledge, albeit with less precision than other gauge terms. It encodes two interactions, $H W W$ and $H Z Z$, both of which contributed to the Higgs-boson discovery and continue to see extensive study [2]. In a sense, this part is a variation on the other well-established gauge terms of the Standard Model Lagrangian. 

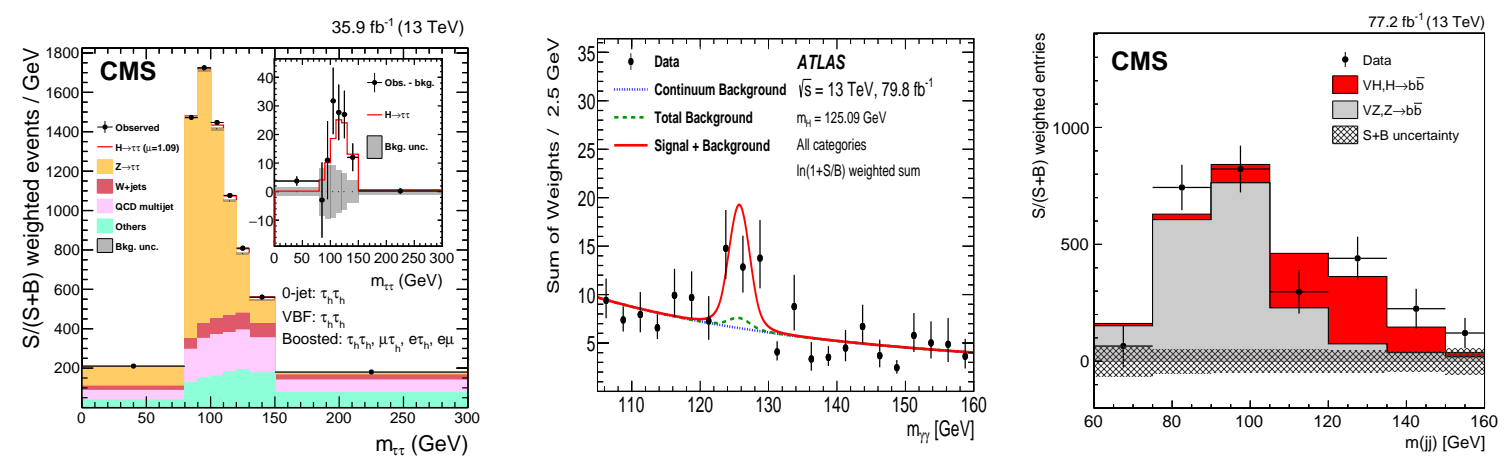

Figure 1: Selection of plots illustrating the observation of $H \rightarrow \tau \tau$ (left), the $t \bar{t} H$ process (middle) and $H \rightarrow b \bar{b}$ (right) by the ATLAS $[6,8,9]$ and CMS collaborations $[5,7,10]$.

The last two terms of Eq. (1) are, in contrast, unlike any fundamental interaction that had been probed before the Higgs boson discovery. Let us first discuss the Yukawa term.

\subsection{The Higgs Yukawa sector}

Within the Standard Model hypothesis, the Higgs Yukawa term, $\psi_{i} y_{i j} \psi_{j} \phi$, generates masses for all quarks and charged leptons. Experimentally, the hypothesis that the Higgs field genuinely produces these mass terms can be tested by probing $H f f$ type interactions, where $f$ is any massive fermion, and verifying the proportionality of interaction in the amplitude with the fermion mass. Considering a flavour basis in which the $y_{i j}$ are diagonal, there are nine independent terms (though one should also check for flavour changing Higgs interactions, $H f f^{\prime}$ ).

Prior to the discovery of the Higgs boson there was no evidence for fundamental Yukawa interactions: this was not the part of the Standard Model that had been probed by 40 years of tests, not even indirectly at LEP. Discovery provided indirect evidence for two of the nine interactions. Specifically, the consistency of the cross section in all observed decay channels was both sensitive to, and consistent with, the Standard Model expectation for the top and bottom Higgs interactions, given that the $H t t$ coupling appears in the $g g \rightarrow H$ and $H \rightarrow \gamma \gamma$ effective interactions, while the $\mathrm{Hbb}$ coupling dominates the overall width of the Higgs boson and so affects all branching fractions and cross sections.

Over the past 18 months, our knowledge of Higgs Yukawa interactions has undergone a revolution, with all three of the (charged-fermion) third-generation Yukawa couplings now established directly at $5 \sigma$, independently by each of the ATLAS and CMS collaborations, through the observation Higgs decays to $\tau^{+} \tau^{-}[5,6]$, Higgs production in association with a $t \bar{t}$ system $[7,8]$ and Higgs decays to $b \bar{b}[9,10]$. A selection of corresponding plots is shown in Fig. 1. ${ }^{1}$ This part of the Standard Model is no longer a hypothesis. It is quite clearly a fact, at least to within the roughly $20 \%$ accuracy that accompanies a $5 \sigma$ discovery.

What importance should we, as a field, attribute to the observation of Yukawa interactions? I would argue that it is comparable to the importance of the discovery of the Higgs boson in the first place, for three main reasons.

\footnotetext{
${ }^{1}$ When this talk was originally given, only the top and $\tau$ couplings had been established.
} 
The first reason is that it is exceedingly rare to observe a qualitatively new kind of interaction. At the risk of oversimplification, so far matter particles, fermions, were known to have gravity and gauge interactions; now we know that they have a third kind, Yukawa scalar interactions.

The second reason is related to the major role that (still hypothetical) first-generation Yukawa interactions play in the world we experience every day. One example concerns the proton-neutron mass difference. The proton is lighter than the neutron, despite the fact that it is electrically charged while the neutron is not. As quantified for example in Ref. [11] this is a consequence of the down quark mass (about $4.7 \mathrm{MeV}$ ) being larger than the up quark mass (about 2.2 MeV). Within the Standard Model hypothesis, that stems from the pattern of first-generation Yukawa couplings. Were the first generation quark Yukawa couplings to be zero, leading to correspondingly massless quarks, protons would be unstable because they would be heavier than neutrons. As a result there would be no stable hydrogen. ${ }^{2}$ The electron's Yukawa coupling is equally important: through its impact on the electron mass it sets the size of atoms and their energy levels, the Bohr radius being proportional to $1 / m_{e} .^{3}$

A third reason for assigning importance to the observation of Yukawa couplings is that the mystery of the hierarchy of (charged) fermion masses is, in the Standard Model hypothesis, converted to a mystery about a hierarchy of couplings. Does it matter which of these two we talk about? My attitude is that it would be good to know, for sure, that we are indeed dealing with a hierarchy of couplings. Only then can we be sure of what deeper problem we should be trying to understand. ${ }^{4}$

When communicating with our colleagues in neighbouring fields, and with the general public, we should be wary of talking of vindication of the standard model and an ensuing dead-end [15]. What we could instead be saying about recent developments is:

The $>5 \sigma$ observations of the $t t H$ process and $H \rightarrow \tau^{+} \tau^{-}$and $H \rightarrow b \bar{b}$ decays, independently by ATLAS and CMS, firmly establish the existence a new kind of fundamental interaction, Yukawa interactions.

Yukawa interactions are important not merely because they had never before been directly observed, but also because they are hypothesised to be responsible for the stability of hydrogen, and for determining the size of atoms and the energy scales of chemical reactions. Establishing the pattern of Yukawa couplings across the full remaining set of quarks and charged leptons is one of the major challenges for particle physics today.

The second paragraph of that statement serves as a reminder of just how much more remains to be established before we can truly talk about the vindication of the Standard Model, even just its

\footnotetext{
${ }^{2}$ Deuterium would conceivably still be stable.

${ }^{3}$ A variety of similar thought experiments, specifically about a world without a Higgs field, were carried out by Quigg and Shrock [12]. In contrast to the discussion here, their scenarios have an impact also on the electroweak vector bosons.

${ }^{4}$ To take a simple alternative, the Giudice-Lebedev mechanism [13] generates small first and second generation masses by terms in the Lagrangian $Y_{i j}(\phi) \psi_{i} \psi_{j} \phi+$ h.c. with $Y_{i j}(\phi)=c_{i j}\left(\phi^{\dagger} \phi / M^{2}\right)^{n_{i j}}$, where $M$ is some large new mass scale, which would predict enhancement of apparent Higgs-fermion interaction strengths (in the amplitude) by a factor $2 n_{i j}+1$. A more sophisticated realisation of this picture, taking into account recent constraints, is discussed in Ref. [14]. I am grateful to Uli Haisch for discussions on these points.
} 

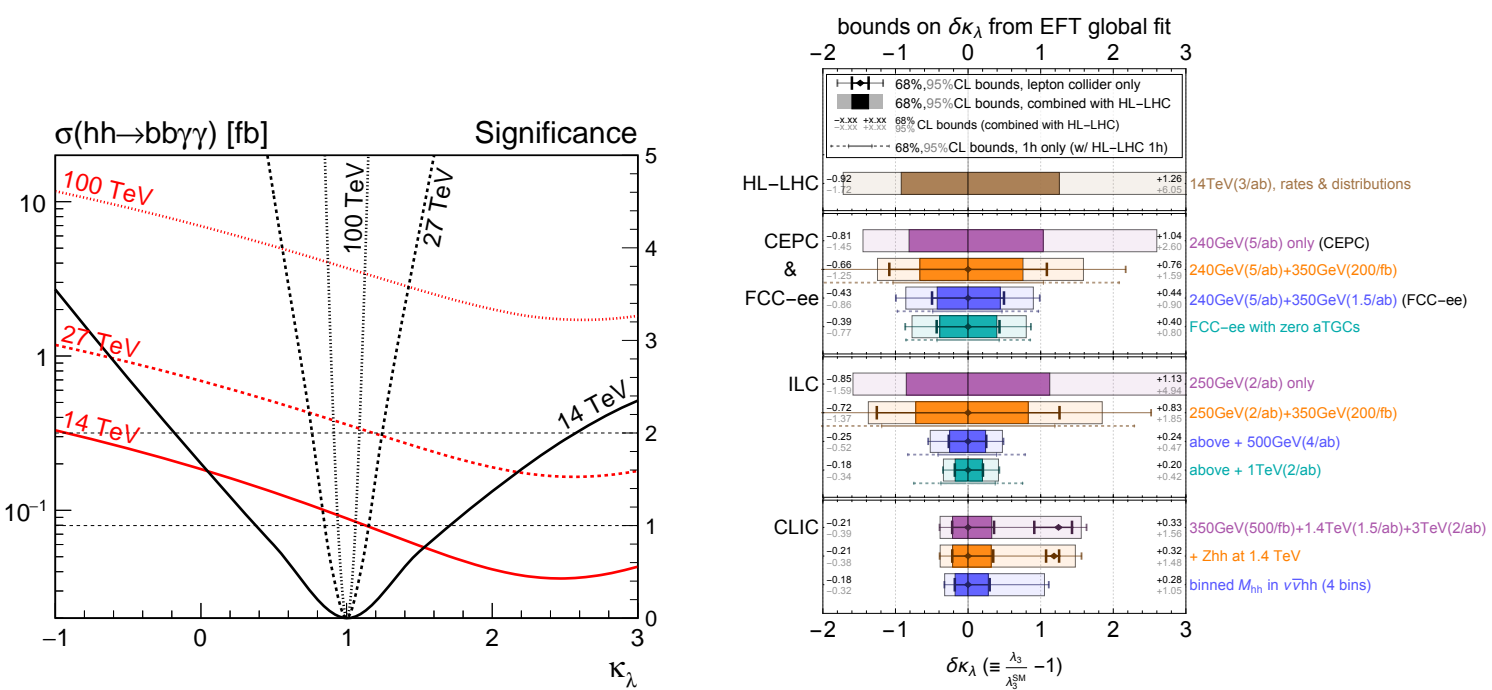

Figure 2: Projected constraints on the coefficient $\lambda$ (or rather the factor $\kappa_{\lambda}$ by which it deviates from the Standard Model) of the triple-Higgs interaction at a variety of future colliders (this is not the same $\lambda$ that appears in Eq. (1)). Left: at hadron colliders [20], showing the cross section in red and the contour of constraint significance in black (see also Ref. [21]). Right: at lepton colliders [22], compared to the HLLHC projection.

Yukawa sector. In some cases we know how to go about learning more. For example, the muon Yukawa coupling can be established at the HL-LHC, through observation of $H \rightarrow \mu^{+} \mu^{-}$decays. Establishing the charm Yukawa coupling probably requires a lepton collider (see e.g. the analysis for the ILC [16]), or an $e p$ facility [17]. Importantly, the total width can also be strongly constrained at $e^{+} e^{-}$colliders. For other Yukawa couplings, the path forwards still remains to be established: ideas for constraining the quark Yukawa sector are highlighted in the references of Refs. [14, 18] and it has even been suggested that the electron Yukawa coupling could be probed in $s$-channel resonant Higgs production at the FCC-ee [19].

\subsection{The Higgs self coupling}

Aside from the Yukawa interactions, the other qualitatively new structure in Eq. (1) is the Higgs potential term, $V(\phi)=-\mu^{2} \phi^{2}+\lambda \phi^{4}$, which generates $H H H$ interactions. A theory with $\phi^{4}$ interactions is familiar insofar as scalar theory with $\phi^{n}$ interactions serves for almost every graduate student's introduction to field theory. Yet we should not forget that a fundamental $\phi^{4}$ theory has never been observed in nature. Nor is there even any indirect experimental evidence for it. This part of the Standard Model is not merely not yet knowledge, the non-trivial part of it, i.e. structure beyond the simple existence of a minimum and associated vacuum expectation value, is totally unexplored. It is also the keystone of the Standard Model: without the potential, the rest of the edifice falls down.

Together with the Yukawa couplings, determining as much of the potential as we can should, consequently, be one of the major targets for the field. Projected constraints from a range of colliders on the triple-Higgs interaction strength, which is directly correlated with the structure of the Higgs potential, are shown in Fig. 2. 

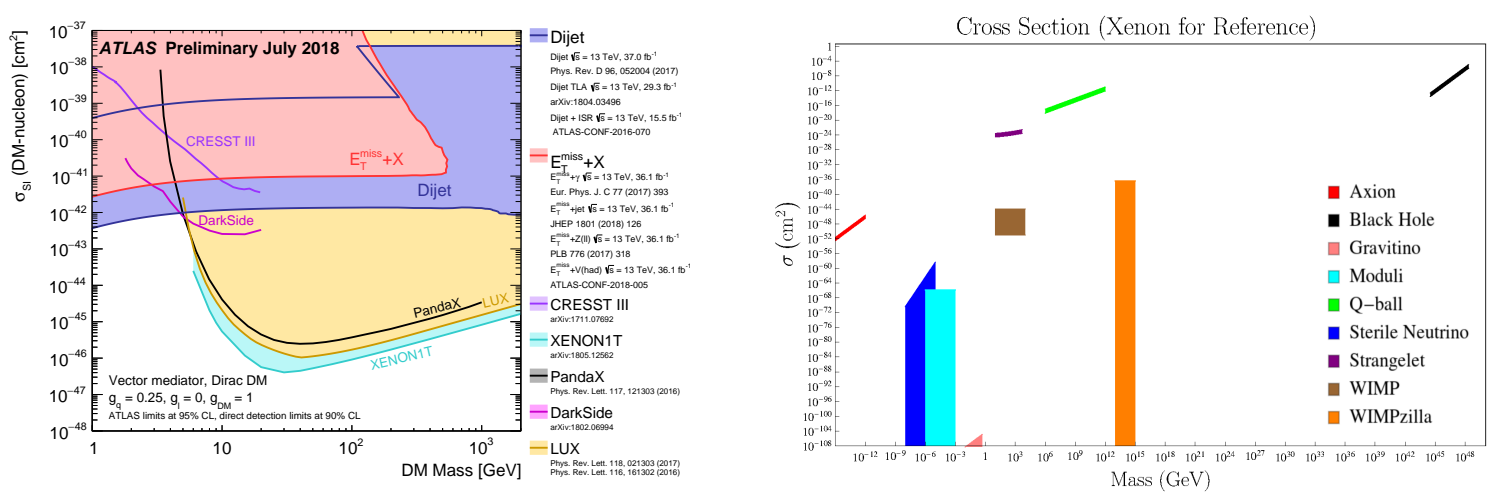

Figure 3: Left: dark-matter exclusion summary plot [25]. The comparison between limits from different experiments is model dependent. Right: the direct-detection cross-section versus mass plane for a set of dark-matter models, taken from a Snowmass report [26].

\subsection{Higgs outlook}

Overall it seems clear that if we are to push our knowledge of the Higgs sector to as much of the Lagrangian as is possible, and with reasonable precision, then in addition to the progress that is expected to come from the HL-LHC we will need a lepton collider and a higher-energy hadron collider.

One question whose answer is not yet clear to me, is the precision that we should seek on our determinations of Higgs interactions, both the self-coupling and the others. A threshold of $\sim 20 \%$ (on a squared interaction) is akin to the $5 \sigma$ condition for declaring "observation" of a new particles, and seems a minimal requirement. Once an interaction is established, then should one aim to go the percent level, to test quantum effects? ${ }^{5}$ Should one aim to test the coupling over, e.g., an order of magnitude in transverse momentum, to gain confidence that it is not contaminated by large higher-dimension operators? Should models of new physics and the nature of the electroweak phase transition act as a guide in such circumstances? Or should we simply set out to measure all fundamental properties of our universe to some basic precision, say $1 \%$, independently of models for new physics? These are questions that I believe we as a community need to discuss, and they feed into the choices that we will argue for as to the next machine(s) to support.

\section{Searches and anomalies}

Prior to the first run of the LHC, many physicists made statements that they expected the discovery of supersymmetry (e.g. Ref. [23]). At the start of Run 2, with supersymmetry, and alternatives such as extra dimensions, proving elusive, some of the emphasis had shifted to dark matter, especially in communication with the public (e.g. [24]). Yet, so far, neither has been found, whether at colliders or elsewhere, cf. e.g. the left-hand plot of Fig. 3. As already alluded to above, this situation is fostering a quite widespread sense of dead-end and crisis.

For some time, I have felt that we ought to be more cautious in our statements of what we expect to find (even if, on occasion, I too have made statements along similar lines), and of the

\footnotetext{
${ }^{5}$ A suggestion made to me by M. McCullough.
} 


\begin{tabular}{ccc}
\hline $\begin{array}{c}\text { LHCP 2018 } \\
\text { reach } \\
\left(13{\left.\mathrm{TeV} 80 \mathrm{fb}^{-1}\right)}^{2}\right.\end{array}$ & $\begin{array}{c}\text { estimated } \\
\text { HL-LHC reach } \\
\left(14 \mathrm{TeV}, 3 \mathrm{ab}^{-1}\right)\end{array}$ & $\begin{array}{c}\text { energy needed } \\
\text { for same reach } \\
\text { with } 80 \mathrm{fb}^{-1}\end{array}$ \\
\hline $\begin{array}{c}4.7 \mathrm{TeV} \mathrm{SSM} Z^{\prime} \\
2 \mathrm{TeV} \text { weakly } \\
\text { coupled } Z^{\prime} \\
680 \mathrm{GeV}\end{array}$ & $6.7 \mathrm{TeV}$ & $20 \mathrm{TeV}$ \\
chargino & $1.7 \mathrm{TeV}$ & $37 \mathrm{TeV}$ \\
\hline
\end{tabular}

Table 1: Rough ATLAS/CMS exclusion sensitivities at the time of the conference, extrapolation to the HL-LHC, and estimate of the corresponding collider centreof-mass energy that would be needed to obtain the same reach if one were to increase the collider energy while keeping a fixed luminosity. (HL-LHC and highenergy collider estimates made using the collider-reach tool [29].)

extent of the crisis that comes from not having discovered them. One of the most appealing elements about the fine-tuning argument in favour of supersymmetry was that such arguments had worked before, for example in the prediction of the charm quark. Yet in any prediction by theorists that is not an actual robust proof, one should allow for the possibility that a new idea could change our perspective on the classes of solution that might exist for the underlying problem that they are trying to address, as in some sense has happened with fine tuning [27]. ${ }^{6}$ As for dark matter, the parameter space and variety of models is vast, as illustrated in Fig. 3 (right).

The point of view that I would advocate is that, as a field, we are trying to address problems that have a long history of being difficult to solve. For example, the evidence for dark matter dates back to the 1930's. We may hold out hope that a given new run of the LHC or of a direct detection experiment will discover it. It is natural to be disappointed if that turns out not to happen. However, we should not be overly surprised if solutions to such long-standing problems don't fall into our lap each time a new machine is turned on. Perhaps, instead, it should serve as a reminder that the immense challenge of solving such problems is an intrinsic part of the fascination of the field.

Perhaps another contributing factor to the feeling of crisis comes from the fact that LHC has now been operating at close to design energy for a few years and that "all" that is left is an increase in luminosity. Yet the power of luminosity should not be underestimated, even aside from the immense progress that it will bring in Higgs-sector studies. Table 1 shows estimates of HL-LHC (exclusion) sensitivity based on extrapolations from the reach at the time of the conference. It also shows what energy collider would be needed to obtain the same reach by increasing the centreof-mass energy rather than the luminosity. The answer depends significantly on the mass region that is already being probed. The chargino example is notable, where the equivalent centre-of mass energy is over four times the current LHC energy: increased luminosity buys the field substantial reach.

Still, the field will have to contend with the fact that over the next twenty years the rate of logarithmic integrated luminosity increase at the LHC will be considerably slower than it has been so far. In such a context, the reduced pressure to update results for each new conference can perhaps be compensated by further encouraging opportunities for creative thinking, whether for novel kinds

\footnotetext{
${ }^{6}$ Incidentally, even had supersymmetry been discovered, it is not inconceivable that some people would still have claimed that the field is in a crisis. As far back as 1995, statements were being made such as "in the MSSM we have nothing but boring perturbative physics to explore below the Planck scale and the interesting dynamics of supersymmetry breaking is hidden." [28].
} 
of searches (for example long-lived particle searches [30]), new ways of incorporating machine learning, approaches to automating searches (e.g. [31]), or even finding ways of making existing LHC data more accessible to researchers who are not members of a given experiment [32]. ${ }^{7}$

This section wouldn't be complete without a mention of a number of anomalies across different sectors of particle physics: those in the flavour sector saw much attention at this conference (see Hiller and Humair's talks [34, 35]). Others include the longstanding issue in $g_{\mu}-2$ [36], short baseline neutrino experiment anomalies, e.g. as recently seen by Miniboone [37], the $16.7 \mathrm{MeV}$ Beryllium 8 peak [38], the hint of a di-muon peak in events with a $b \bar{b}$ in ALEPH and CMS [39, 40]. One may still be optimistic that one or other of them will, in the coming years, see the corroborating evidence needed to establish the existence of laboratory-accessible physics beyond the standard model (aside from the existence of neutrino masses).

Going forwards, a question that I feel we ought to discuss more is that of how we prioritise searches in different regions of parameter space. At the LHC, this is relatively straightforward: one searches for everything that can be searched for. But beyond the LHC, should one evaluate an experiment simply based on the new area in the $\ln$ (coupling)- $\ln$ (mass) plane that it will cover, multiplied by the number of channels? Should one prioritise high mass scales, whether on finetuning grounds (there is still a chance that this is the right perspective), or because most of our discoveries so far have come from exploring ever higher scales?

\section{Precision physics at the LHC}

One of the surprises with the LHC is its remarkable potential for precision measurements. These include improved determinations of fundamental parameters such as the $W$ and top-quark masses, constraints on parton distribution functions, precise evaluations of backgrounds for missing energy searches, and more generally constraints on higher-dimensional operators. In due course, Higgs physics will also join the precision family.

Precision matters in its own right: the confidence that we have in a hypothesis that is supported by experimental results to within $1 \%$ precision is very different from a situation where we are limited to $20 \%$ tests. Additionally, precision can contribute significantly to sensitivity to physics beyond the Standard Model. As an example, considering constraints on the energy-scale of dimension-6 operators (in processes that involve interference with Standard Model operators), statistically those constraints scale as the fourth root of the luminosity, $\Lambda_{\text {dim- } 6} \sim \mathcal{L}^{\frac{1}{4}}$. Going from $80 \mathrm{fb}^{-1}$ to $3 \mathrm{ab}^{-1}$ could then translate to an increase in mass scale sensitivity by a factor of almost 2.5 , which is greater than any of the estimates for direct searches shown in table 1 .

An important question for the future of precision physics at the LHC is our understanding of, and ability to make progress in reducing systematic uncertainties, both experimental and theoreti-

\footnotetext{
${ }^{7}$ Straightforward access to research-usable open data clearly remains a challenge for the field, even if some progress has been made in the past couple of years by CMS (with the data also being used by theorists [33]). Perhaps one step towards encouraging progress would be for major conferences such as LHCP to schedule a dedicated parallel session, and associated plenary talk, on developments and issues in making research-quality data accessible (the question goes well beyond one of outreach, which the session where the topic is currently addressed). At the very least, this would help give visibility to those involved in this challenging task and communicate the message that the field genuinely values the endeavour.
} 
cal. As an example, Huss and Melnikov's talks [41, 42] at this conference summarised the rapid advances that are being made in calculations of higher-order perturbative QCD and EW contributions to many LHC processes. Prestel's talk at this conference [43] outlined some of the recent progress in general-purpose Monte Carlo event generators. This is an important area because differences between generators are quite often among the dominant systematic in experimental calibrations and measurements. ${ }^{8}$

Two areas that are potential long-term limiting factors are non-perturbative corrections on the theoretical side and the uncertainty in the absolute luminosity on the experimental side (see for example Valentinetti's talk [45]). The former has recently seen a pioneering analysis [46], sensitive to all main aspects of the leading (relative $\Lambda_{\mathrm{QCD}} / m_{\text {top }}$ suppressed) non-perturbative contributions in top-quark measurements, both for cross sections and the reconstructed top mass. Possible avenues of progress for the latter are discussed in Ref. [47].

\section{Heavy-ion collisions}

Over the past twenty years, heavy-ion collisions have revealed an array of new and intriguing phenomena. The latest instalment is the discovery that proton-proton and proton-lead collisions yield signals of collective effects (elliptic flow) that had long been thought to be characteristic of larger systems such as lead-lead. This was summarised in the talk by Bellini [48]. The condition for such signals of collective effects to be observed is that one should focus on the subset of protonproton and proton-lead collisions that have a high final-state multiplicity. Thus the field of heavyion physics is teaching us new ways of looking at, and new features of, proton-proton collisions.

At the same time, techniques originally developed in the context of new-physics searches, in particular jet-substructure techniques [49] are today seeing applications in heavy-ion collisions, with the heavy-ion field being a very fast adopter of the latest developments, as reviewed in the talk by Apolinário [50].

One possibility that has seen first feasibility studies recently is that of using electroweakscale probes in heavy-ion collisions [51] either to learn more about the quark-gluon plasma or the electroweak-scale probes themselves. For example, Ref. [52] has suggested that one may better measure the coupling of the Higgs to $b$-quarks in heavy-ion collisions because the medium will quench background $b$-jets and not those from a (long-lived) Higgs decay to $b \bar{b}$. Ref. [53] has suggested the Higgs width may be significantly modified while passing through the medium, though one should keep in mind that this requires further theoretical analysis, especially given the subtleties that arise in width calculations at finite temperature [54]. ${ }^{9}$ I myself have become interested in the question of whether the finite lifetime of top-quark and $W$ bosons, as well as the finite decoherence time of the $q \bar{q}^{\prime}$ system from $W$ decay could be exploited to give time-resolved information about the quark-gluon plasma [55]. In all cases, given the small cross sections of electroweakscale probes, it seems likely that increased luminosity and/or collider energy are a necessity for these studies to become reality. The possibility of obtaining higher nucleon-nucleon luminosities in collisions of intermediate-size systems [56] may be important in this respect.

\footnotetext{
${ }^{8}$ I am perhaps biased here, having recently developed an active interest in the topic of what parton shower algorithms can and should achieve [44].

${ }^{9}$ I am grateful to Urs Wiedemann for pointing out this reference to me.
} 


\section{Conclusions}

Many in our field talk of a period of crisis because the LHC has "failed" to discover physics beyond the Standard Model. I would argue that we might equally think of this as a golden period of particle physics, in particular from an experimental point of view: it is not often that physicists gain access to new, apparently fundamental classes of interaction, and that is precisely what the discovery of the Higgs boson has made possible for us, in the past 18 months with the observation of the third-generation Yukawa interactions, in the future with Yukawa couplings beyond the 3rd generation and the $\phi^{4}$ structure of the Higgs potential.

Of course, the interactions that are being discovered today are far from novel from a theoretical point of view, and this is a cause of despondency for many. Yet, while it is perfectly legitimate to get excited about conceptually new realisations of how our universe might be configured, we should not fall into the trap of failing to be excited when we are in the process discovering how essential aspects of our universe actually are configured. The fact that these aspects were hypothesised long ago does not take away from their importance. On the contrary, even if we are still left with big unanswered questions such as the nature of dark matter and the solution to the hierarchy problem, we should retain some capacity for wonder and awe at the fact that what is arguably the minimal hypothesis - the Standard Model — actually seems, for now, to be how the directly accessible part of nature is arranged.

\section{Acknowledgements}

I am grateful to Uli Haisch, Matthew McCullough, Michelangelo Mangano, Guilherme Milhano, Hitoshi Murayama, Urs Wiedemann and Giulia Zanderighi for stimulating conversations on topics related to this writeup and/or for helpful comments. This work is supported in part by a Royal Society Research Professorship under grant RP $\backslash R 1 \backslash 180112$.

\section{References}

[1] C. Grefe, "Higgs couplings to fermions at the ATLAS and CMS experiments", this conference.

[2] G. Ortona, "Measurements in Higgs decays to diboson final states at the ATLAS and CMS experiments", this conference.

[3] V. Sanz, "Effective field theory and the Higgs", this conference.

[4] E. Vryonidou, "Theoretical implications of the $125 \mathrm{GeV}$ Higgs boson", this conference.

[5] A. M. Sirunyan et al. [CMS Collaboration], "Observation of the Higgs boson decay to a pair of $\tau$ leptons with the CMS detector”, Phys. Lett. B 779 (2018) 283 [arXiv:1708.00373 [hep-ex]].

[6] The ATLAS collaboration [ATLAS Collaboration], "Cross-section measurements of the Higgs boson decaying to a pair of tau leptons in proton-proton collisions at $\sqrt{s}=13 \mathrm{TeV}$ with the ATLAS detector", ATLAS-CONF-2018-021.

[7] A. M. Sirunyan et al. [CMS Collaboration], "Observation of tẗH production", Phys. Rev. Lett. 120 (2018) no.23, 231801 [arXiv:1804.02610 [hep-ex]]. 
[8] M. Aaboud et al. [ATLAS Collaboration], "Observation of Higgs boson production in association with a top quark pair at the LHC with the ATLAS detector", Phys. Lett. B 784 (2018) 173 [arXiv:1806.00425 [hep-ex]].

[9] M. Aaboud et al. [ATLAS Collaboration], "Observation of $H \rightarrow b \bar{b}$ decays and $V H$ production with the ATLAS detector”, Phys. Lett. B 786 (2018) 59 [arXiv:1808.08238 [hep-ex]].

[10] A. M. Sirunyan et al. [CMS Collaboration], "Observation of Higgs boson decay to bottom quarks", Phys. Rev. Lett. 121 (2018) no.12, 121801 [arXiv:1808.08242 [hep-ex]].

[11] S. Borsanyi et al. [Budapest-Marseille-Wuppertal Collaboration], "Isospin splittings in the light baryon octet from lattice QCD and QED”, Phys. Rev. Lett. 111 (2013) no.25, 252001 [arXiv:1306.2287 [hep-lat]].

[12] C. Quigg and R. Shrock, "Gedanken Worlds without Higgs: QCD-Induced Electroweak Symmetry Breaking”, Phys. Rev. D 79 (2009) 096002 [arXiv:0901.3958 [hep-ph]].

[13] G. F. Giudice and O. Lebedev, "Higgs-dependent Yukawa couplings", Phys. Lett. B 665 (2008) 79 [arXiv:0804.1753 [hep-ph]].

[14] M. Bauer, M. Carena and A. Carmona, "Higgs Pair Production as a Signal of Enhanced Yukawa Couplings”, Phys. Rev. Lett. 121 (2018) no.2, 021801 [arXiv:1801.00363 [hep-ph]].

[15] A. Frank and M. Gleiser, "A Crisis at the Edge of Physics", 5 June 2015 in the New York Times, https://www .nytimes.com/2015/06/07/opinion/a-crisis-at-the-edge-of-physics.html.

[16] K. Fujii et al., "Physics Case for the $250 \mathrm{GeV}$ Stage of the International Linear Collider", arXiv:1710.07621 [hep-ex].

[17] M. Klein, “Future Deep Inelastic Scattering with the LHeC”, arXiv:1802.04317 [hep-ph].

[18] J. Duarte-Campderros, G. Perez, M. Schlaffer and A. Soffer, "Probing the strange Higgs coupling at lepton colliders using light-jet flavor tagging”, arXiv:1811.09636 [hep-ph].

[19] D. d'Enterria, "Higgs physics at the Future Circular Collider", PoS ICHEP 2016 (2017) 434 [arXiv:1701.02663 [hep-ex]].

[20] D. Gonçalves, T. Han, F. Kling, T. Plehn and M. Takeuchi, "Higgs boson pair production at future hadron colliders: From kinematics to dynamics”, Phys. Rev. D 97 (2018) no.11, 113004 [arXiv:1802.04319 [hep-ph]].

[21] R. Contino et al., "Physics at a 100 TeV pp collider: Higgs and EW symmetry breaking studies", CERN Yellow Report (2017) no.3, 255 [arXiv:1606.09408 [hep-ph]].

[22] S. Di Vita, G. Durieux, C. Grojean, J. Gu, Z. Liu, G. Panico, M. Riembau and T. Vantalon, "A global view on the Higgs self-coupling at lepton colliders", JHEP 1802 (2018) 178 [arXiv:1711.03978 [hep-ph]].

[23] P. Catapano, "Nobel expectations for new physics at the LHC", CERN Courier, 19 August 2008, https://cerncourier.com/nobel-expectations-for-new-physics-at-the-lhc/

[24] PBS, "Large Hadron Collider gears up to find dark matter, new particles in its second run", 12 March 2016, https://www . pbs.org/newshour/science/large-hadron-collider-gears-find-dark- $\neg$ matter-new-particles-second-run.

[25] ATLAS Collaboration, Dark Matter summary plots, version of July 2018, https://atlas.web.cern.ch/Atlas/GROUPS/PHYSICS/CombinedSummaryPlots/EXOTICS/ATLAS_ᄀ DarkMatter_ZCrossSection_VectorNucleon/history.html 
[26] A. Kusenko and L. J. Rosenberg, "Working Group Report: Non-WIMP Dark Matter", arXiv:1310.8642 [hep-ph].

[27] P. W. Graham, D. E. Kaplan and S. Rajendran, "Cosmological Relaxation of the Electroweak Scale", Phys. Rev. Lett. 115 (2015) no.22, 221801 [arXiv:1504.07551 [hep-ph]].

[28] A. E. Nelson, "Consequences of low-energy dynamical supersymmetry breaking", In Tahoe City 1994, Proceedings, Beyond the standard model 4, 396-398 [hep-ph/9503345].

[29] G. P. Salam and A. Weiler, "Collider Reach", http://cern.ch/collider-reach.

[30] J. Michael Williams, "Searches for unconventional and long-lived signatures", this conference.

[31] M. Aaboud et al. [ATLAS Collaboration], "A strategy for a general search for new phenomena using data-derived signal regions and its application within the ATLAS experiment", [arXiv:1807.07447 [hep-ex]].

[32] L. Lloret Iglesias, "Open data at the LHC", this conference.

[33] A. Larkoski, S. Marzani, J. Thaler, A. Tripathee and W. Xue, "Exposing the QCD Splitting Function with CMS Open Data", Phys. Rev. Lett. 119 (2017) no.13, 132003 [arXiv:1704.05066 [hep-ph]].

[34] G. Hiller, "Theoretical implications of flavour anomalies", this conference.

[35] T. Humair, "Lepton flavour universality tests and lepton flavour violation searches", this conference.

[36] A. E. Dorokhov, A. E. Radzhabov and A. S. Zhevlakov, "The muon g-2: retrospective and future", EPJ Web Conf. 125 (2016) 02007 [arXiv:1608.02331 [hep-ph]].

[37] A. A. Aguilar-Arevalo et al. [MiniBooNE Collaboration], "Significant Excess of ElectronLike Events in the MiniBooNE Short-Baseline Neutrino Experiment", arXiv:1805.12028 [hep-ex].

[38] A. J. Krasznahorkay et al., "Observation of Anomalous Internal Pair Creation in Be8 : A Possible Indication of a Light, Neutral Boson”, Phys. Rev. Lett. 116 (2016) no.4, 042501 [arXiv:1504.01527 [nucl-ex]].

[39] A. Heister, "Observation of an excess at $30 \mathrm{GeV}$ in the opposite sign di-muon spectra of $\mathrm{Z} \rightarrow b \bar{b}+\mathrm{X}$ events recorded by the ALEPH experiment at LEP", arXiv:1610.06536 [hep-ex].

[40] A. M. Sirunyan et al. [CMS Collaboration], "Search for resonances in the mass spectrum of muon pairs produced in association with b quark jets in proton-proton collisions at $\sqrt{s}=8$ and $13 \mathrm{TeV}$ ", arXiv: 1808.01890 [hep-ex].

[41] A. Huss, "EWK precision physics", this conference.

[42] K. Melnikov, "Frontier precision calculations", this conference.

[43] S. Prestel, "Recent developments in MC", this conference.

[44] M. Dasgupta, F. A. Dreyer, K. Hamilton, P. F. Monni and G. P. Salam, "Logarithmic accuracy of parton showers: a fixed-order study", JHEP 1809 (2018) 033 [arXiv:1805.09327 [hep-ph]].

[45] S. Valentinetti, "Luminosity Measurements", this conference.

[46] S. Ferrario Ravasio, P. Nason and C. Oleari, "All-orders behaviour and renormalons in top-mass observables", arXiv:1810.10931 [hep-ph].

[47] A. Dabrowski, "Luminosity determination at HL-LHC: a first discussion", Workshop on the physics of HL-LHC, and perspectives at HE-LHC, 18-20 June 2018, CERN, Geneva.

https://indico.cern.ch/event/686494/contributions/2984655/attachments/1670448/2679557ᄀ /HL-LHC_Workshop_Luminosity_Precision_Perspectives_Experiments_19_06_2018.pdf 
[48] F. Bellini, "From small to large colliding systems: lessons learned and future perspectives", this conference.

[49] A. J. Larkoski, I. Moult and B. Nachman, "Jet Substructure at the Large Hadron Collider: A Review of Recent Advances in Theory and Machine Learning", arXiv:1709.04464 [hep-ph].

[50] L. Apolinário, "Overview of jet quenching and energy loss in heavy-ion collisions", this conference.

[51] D. d'Enterria, "Top-quark and Higgs boson perspectives at heavy-ion colliders", Nucl. Part. Phys. Proc. 289-290 (2017) 237 [arXiv:1701.08047 [hep-ex]].

[52] E. L. Berger, J. Gao, A. Jueid and H. Zhang, "Higgs properties revealed through jet quenching in heavy ion collisions", arXiv:1804.06858 [hep-ph].

[53] D. d'Enterria and C. Loizides, "Higgs boson suppression in quark-gluon matter", arXiv:1809.06832 [hep-ph].

[54] A. Czarnecki, M. Kamionkowski, S. K. Lee and K. Melnikov, "Charged Particle Decay at Finite Temperature", Phys. Rev. D 85 (2012) 025018 [arXiv:1110.2171 [hep-ph]].

[55] L. Apolinário, J. G. Milhano, G. P. Salam and C. A. Salgado, "Probing the time structure of the quark-gluon plasma with top quarks", Phys. Rev. Lett. 120 (2018) no.23, 232301 [arXiv:1711.03105 [hep-ph]].

[56] J. M. Jowett, "Colliding heavy ions beyond run 2", talk at Workshop on the physics of HL-LHC, and perspectives at HE-LHC (2017), https://indico . cern. ch/event/647676/contributions/2721134ᄀ /attachments/1548951/2432673/HL-HE-LHC_physics_Jowett_300ct2017.pdf. 